\title{
Requirement of the combination of mushroom body $\gamma$ lobe and $\alpha / \beta$ lobes for the retrieval of both aversive and appetitive early memories in Drosophila
}

\author{
Zhiyong Xie, ${ }^{1}$ Cheng Huang, ${ }^{1,2}{ }^{B}$ o Ci, ${ }^{1}$ Lianzhang Wang, ${ }^{1}$ and Yi Zhong ${ }^{1,3,4}$ \\ ${ }^{1}$ School of Life Sciences, Tsinghua University, Beijing, 100084, China; ${ }^{2}$ Department of Biological Sciences, Stanford University, \\ Stanford, California 94305, USA; ${ }^{3}$ Cold Spring Harbor Laboratory, Cold Spring Harbor, New York 11724, USA
}

\begin{abstract}
Extensive studies of Drosophila mushroom body in formation and retrieval of olfactory memories allow us to delineate the functional logic for memory storage and retrieval. Currently, there is a questionable disassociation of circuits for memory storage and retrieval during Drosophila olfactory memory processing. Formation of the initial aversive olfactory memory involves mushroom body $\gamma$ lobe, whereas $\alpha / \beta$ lobes are reported to be necessary for the retrieval of such memory. In contrast, formation and retrieval of the short-term appetitive olfactory memory appears to involve $\gamma$ lobe. With the help of newly identified Gal4 lines and of focusing on 3-h memory for both aversive and appetitive conditionings, our reexamination of the retrieval of aversive and appetitive olfactory memories suggests a new view. Blocking $\gamma$ lobe output led to severe deficiency of aversive early memory retrieval and partial impairment of appetitive early memory retrieval. Interrupting $\alpha / \beta$ lobe output impaired the retrieval of both aversive and appetitive early memories. The contribution of the $\gamma$ lobe and $\alpha / \beta$ lobes appeared to be additive for the retrieval of appetitive early memory. Thus, these results suggest that the retrieval of aversive and appetitive olfactory early memories requires the synaptic outputs from both $\gamma$ lobe and $\alpha / \beta$ lobe neurons. This discovery may help us to rethink how aversive and appetitive memories are processed from memory formation to memory retrieval.
\end{abstract}

[Supplemental material is available for this article.]

Characterizing the neural circuits involved in memory retrieval is crucial for understanding memory processing within an intact neural circuit (Silva et al. 2009; van Strien et al. 2009), as well as the pathological basis of various neurological diseases associated with memory deficiency (Kuhlmann et al. 2005; Small et al. 2011; Tye and Deisseroth 2012). Drosophila memory studies have allowed impressive progress in elucidating functional neural circuits and evolutionarily conserved molecular basis underlying memory processing (Davis 2005; Margulies et al. 2005; Keene and Waddell 2007; Bellen et al. 2010; Zars 2010).

Drosophila can associate odor stimulus with electric-shock punishment or sugar reward to form aversive or appetitive olfactory memory (Tempel et al. 1983; Tully and Quinn 1985). Mushroom body $(\mathrm{MB})$ plays a central role in olfactory memory formation and retrieval in Drosophila (Davis 2005; Margulies et al. 2005; Keene and Waddell 2007). It consists of three substructures: $\alpha / \beta$ lobe neurons, $\alpha^{\prime} / \beta^{\prime}$ lobe neurons, and $\gamma$ lobe neurons (Tanaka et al. 2008; Aso et al. 2009). Previous understanding indicates the usage of distinct $\mathrm{MB}$ neural ensembles to retrieve aversive and appetitive early phase memories, including short-term memory (STM) and intermediate-term memory (ITM). $\alpha / \beta$ lobes are reported to be involved in aversive memory retrieval (McGuire et al. 2001; Krashes et al. 2007), while $\gamma$ lobe appears to be necessary for appetitive memory retrieval (Trannoy et al. 2011).

However, such understanding leads to a questionable disassociation of circuits between storage and retrieval of aversive memo-

\section{${ }^{4}$ Corresponding author}

E-mail zhongyi@cshl.edu

Article is online at http://www.learnmem.org/cgi/doi/10.1101//m.031823.113. ry in $\mathrm{MB}$, because previous experiments suggest $\gamma$ lobe, but not $\alpha / \beta$ lobes, is essential for formation of initial aversive memory (Blum et al. 2009; Qin et al. 2012). In MB, appetitive STM formation and retrieval all seem to be confined to $\gamma$ lobe, while $\alpha / \beta$ lobes are dispensable (Trannoy et al. 2011).

In mammalian brain, memory can be retrieved through at least two neuronal ensembles (Takahashi et al. 2008; Goshen et al. 2011; Suzuki and Naya 2011). Since MB shares common origin with vertebrate pallium (Tomer et al. 2010), as well as conserved molecular basis underlying memory processing (Davis 2005; Margulies et al. 2005; Keene and Waddell 2007), it will be meaningful to investigate whether the strategy of using at least two neuronal ensembles to retrieve memory is conserved in Drosophila MB.

To test this hypothesis, the Gal4 lines strongly and preferentially labeling $\gamma$ lobe neurons are needed to examine the roles of $\gamma$ lobe neurons in aversive early memory retrieval (Aso et al. 2009). Stable appetitive memory performance and additional $\alpha / \beta$ lobe Gal4 lines are required to test the role of $\alpha / \beta$ lobe neurons in appetitive early memory retrieval (Trannoy et al. 2011).

We started with screening Gal4 lines reported in the literature, which preferentially label MB $\gamma$ lobe or $\alpha / \beta$ lobe neurons. Using the well-developed genetic tools in Drosophila, we can acutely manipulate the neuronal activity of each MB substructure with good spatial and temporal resolution. Through this approach, we examined the role of each $\mathrm{MB}$ substructure during the retrieval of aversive and appetitive $3-\mathrm{h}$ memories. On this basis, these studies may help us to rethink how aversive and appetitive memories are processed in the example of olfactory learning and memory center in Drosophila. 


\section{Results}

\section{The Gal4 lines used in labeling MB} substructures

$\mathrm{MB} \alpha / \beta$ lobe neurons and olfactory projection neurons can affect the retrieval of olfactory memories (McGuire et al. 2001; Krashes et al. 2007) or the transmission of olfactory information (Masse et al. 2009). So in order to explicitly investigate the roles of $\gamma$ lobe neurons in olfactory conditionings, the Gal4 used to label $\gamma$ lobe should satisfy the following requirements: (1) it has no expression in other MB substructures and olfactory projection neurons; (2) its expression in $\gamma$ lobe is strong; and (3) inhibiting its Gal4 activity specifically in MB should reverse the phenotypes driven by it. Here, we identified two Gal4 lines strongly and preferentially labeling MB $\gamma$ lobe, 5-HT1B-Gal4 (Yuan et al. 2005) and R92F10-Gal4 (Pfeiffer et al. 2008; Jenett et al. 2012), which have not been used in learning and memory studies yet.

We crossed uas-mCD8::GFP;MB247-DsRed flies with each Gal4 line we screened to visualize its expression pattern and its colocalization with MB. MB247-DsRed induces DsRed expression specifically in MB neurons (Lee and Luo 1999; Riemensperger et al. 2005). The expression pattern of mCD8::GFP demonstrated that 5-HT1B-Gal4 strongly and specifically labeled the horizontally located $\gamma$ lobe in $\mathrm{MB}$, without any expression in $\alpha / \beta$ lobes and $\alpha^{\prime} / \beta^{\prime}$ lobes (Fig. 1A) (Yuan et al. 2005). In MB-Gal80;5-HT1B-Gal4 flies, the expression of Gal80 in MB (Lee and Luo 1999; Krashes et al. 2007) specifically inhibited the Gal4 activity of 5-HT1BGal4 in $\gamma$ lobe in $\mathrm{MB}$, while the limited expression of 5-HT1BGal4 in neurons outside MB was not affected (Fig. 1B). Anti-GFP labeling in previous experiments also supports the intensity and preference of 5-HT1B-Gal4 expression in $\gamma$ lobe neurons in $\mathrm{MB}$ (Yuan et al. 2005). R92F10-Gal4 is a new Gal4 line strongly and preferentially labeling MB $\gamma$ lobe neurons (Supplemental Fig. S1; Pfeiffer et al. 2008; Jenett et al. 2012).

$C 739$ and $C 305 a$ are commonly used MB Gal4 lines preferentially expressed in $\alpha / \beta$ lobes and $\alpha^{\prime} / \beta^{\prime}$ lobes in $\mathrm{MB}$, respectively (McGuire et al. 2001; Yu et al. 2006; Krashes et al. 2007; Aso et al. 2009; Blum et al. 2009; Buchanan and Davis 2010; Lee et al. 2011; Huang et al. 2012). NP3061 and NP6024 are another two Gal4 lines preferentially labeling $\mathrm{MB} \alpha / \beta$ lobe neurons (Tanaka et al. 2008; Aso et al. 2009; Huang et al. 2012).

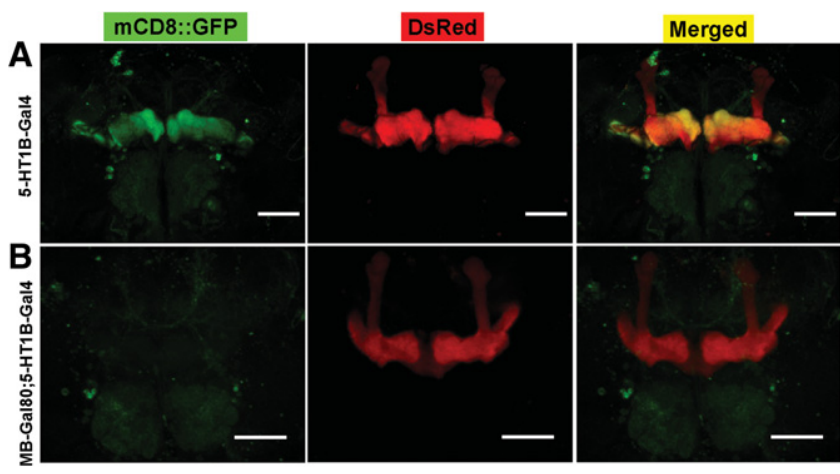

Figure 1. 5-HT1B-Gal4 strongly and preferentially labels $M B \gamma$ lobe neurons. The expression of each Gal4 driver is denoted by the pattern of mCD8::GFP and its colocalization with MB specifically expressed DsRed protein (MB-DsRed). (A) 5-HT1B-Gal4 strongly and restrictively drives the expression of $\mathrm{mCD} 8:$ :GFP in the horizontally located $\gamma$ lobe, without any expression in $\alpha / \beta$ and $\alpha^{\prime} / \beta^{\prime}$ lobes. (B) MB-Gal80;5-HT1BGal4 drives no mCD8::GFP expression in $\gamma$ lobe. The limited expression of $5-H T 1 B-G a l 4$ in the neurons outside $\mathrm{MB}$ is not inhibited by $M B$ Gal80. Scale bar, $50 \mu \mathrm{m}$.
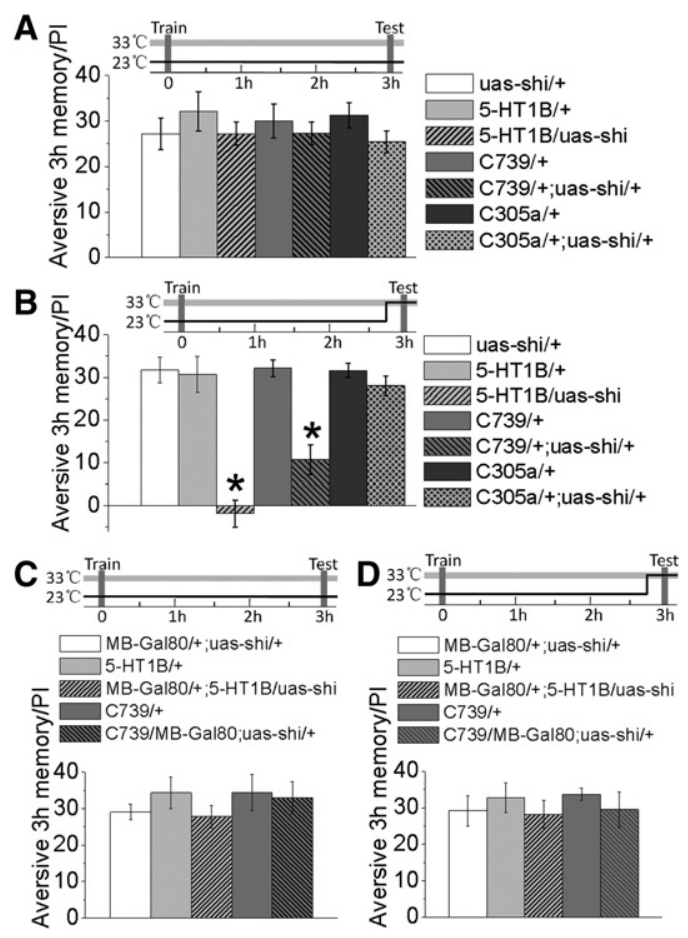

Figure 2. Blocking the output from $\gamma$ lobe or $\alpha / \beta$ lobe neurons impairs the retrieval of aversive 3-h memory. $(A)$ When constantly under the permissive temperature of $23^{\circ} \mathrm{C}$, all genotypes showed normal aversive $3-\mathrm{h}$ memory performances. (B) Blocking the output from 5-HT1B-Gal4 labeled $\gamma$ lobe neurons under the restrictive temperature of $33^{\circ} \mathrm{C}$ severely impaired aversive 3-h memory retrieval. Blocking the output from C739 labeled $\alpha / \beta$ lobe neurons also impaired memory retrieval. Output from C305a labeled $\alpha^{\prime} / \beta^{\prime}$ lobe neurons was dispensable for aversive 3-h memory retrieval. (C) Under the permissive temperature, aversive 3-h memory performance was normal in all genotypes. $(D)$ Inhibiting the Gal4 activity of 5-HT1B-Gal4 or C739-Gal4 in MB by MB-Gal80 reversed the deficiencies of aversive 3-h memory retrieval. In all groups, $N \geq 8$. All error bars indicate SEM. $\left(^{*}\right) P<0.05,(\mathrm{PI})$ performance index.

\section{Blocking the synaptic output from $\gamma$ lobe or $\alpha / \beta$ lobe neurons impairs the retrieval of aversive 3-h memory} Aversive and appetitive 3-h memories formed after one-session training are typical memory phases (intermediate-term memory) for studying different processes of olfactory memory processing, including memory acquisition, consolidation, and retrieval (McGuire et al. 2001; Keene et al. 2006; Krashes et al. 2007; Pitman et al. 2011; Berry et al. 2012).

shibire $^{t s 1}$ is a dominant temperature-sensitive allele of dynamin (Kim and Wu 1990; Kitamoto 2001; Kasuya et al. 2009). Under restrictive temperature, synaptic outputs are acutely blocked in those neurons expressing Shibire ${ }^{\mathrm{ts} 1}$ (Kim and $\mathrm{Wu}$ 1990; Kitamoto 2001; Kasuya et al. 2009). Here, we expressed Shibire $^{\text {ts } 1}$ in each MB substructure driven by different Gal4 lines. Under the permissive temperature of $23^{\circ} \mathrm{C}$, all the genotypes showed normal aversive 3-h memory performances (Fig. 2A,C). When the output from 5-HT1B-Gal4 labeled neurons was blocked under the restrictive temperature of $33^{\circ} \mathrm{C}$, aversive 3 -h memory retrieval was severely impaired (Fig. $2 \mathrm{~B}$ ). Besides, inhibiting the Gal4 activity in MB by MB-Gal80 rescued this memory deficiency (Fig. 2D), which further attested to the role of $\gamma$ lobe in aversive memory retrieval. In addition, 5-HT1B-Gal4/uas-shi and MBGal80/+;5-HT1B-Gal4/uas-shi flies showed normal odor acuity and shock acuity under both permissive and restrictive temperatures (Supplemental Table S1). 

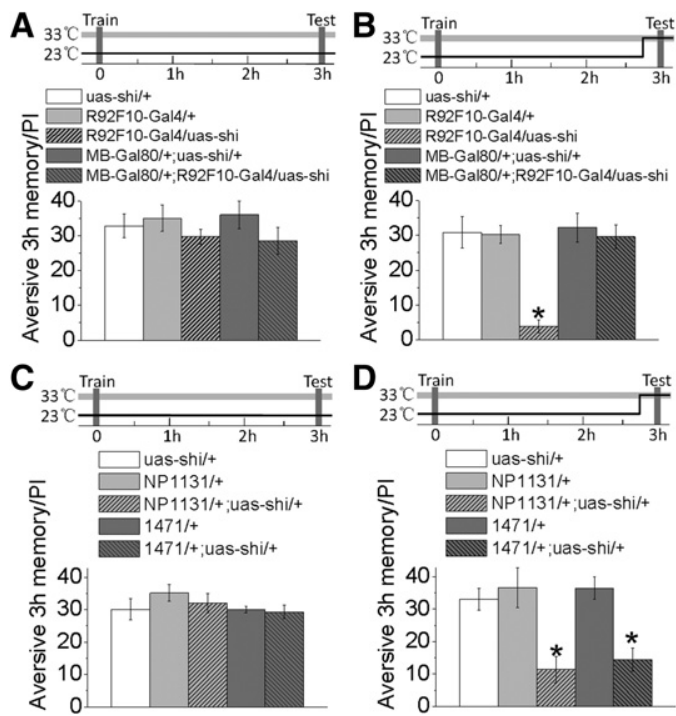

Figure 3. Interrupting the neuronal transmission from the neurons labeled by three other $\gamma$ lobe Gal4s also impairs aversive 3-h memory. (A) Aversive 3-h memory performance was normal in all genotypes under the permissive temperature. (B) Blocking the output from R92F10-Gal4 labeled neurons severely inhibited the retrieval of aversive 3-h memory. Aversive 3-h memory performance was reduced to near zero. Inhibiting the Gal4 activity of R92F10-Gal4 in MB by MB-Gal80 rescued this memory retrieval deficiency. (C) All genotypes had normal aversive 3-h memory performances under the permissive temperature. $(D)$ Aversive 3-h memory was impaired when the neuronal transmission was interrupted in those neurons labeled by another two $\gamma$ lobe Gal4s: NP1131, 1471. NP1131/+;uas-shi/+ and 1471/+;uas-shi/+ flies showed significantly lower memory performance than control groups. In all groups, $N \geq 8$. All error bars indicate SEM. $\left(^{*}\right) P<0.05,(\mathrm{PI})$ performance index.

Consistent with previous studies, blocking the output from $C 739$ labeled $\alpha / \beta$ lobe neurons also impaired aversive 3-h memory retrieval (Fig. 2B,D; McGuire et al. 2001; Krashes et al. 2007). Output from $\alpha^{\prime} / \beta^{\prime}$ lobe neurons was dispensable for aversive 3-h memory retrieval (Fig. 2B; Krashes et al. 2007). In accordance with aversive 3-h memory, blocking the $\gamma$ lobe or $\alpha / \beta$ lobe output also impaired the aversive immediate memory (Supplemental Fig. S2A) and aversive 1-h memory retrieval (Supplemental Fig. S3).

To further testify the role of $\gamma$ lobe in aversive memory retrieval, we also examined another three $\gamma$ lobe Gal4s: R92F10Gal4, NP1131, and 1471.R92F10-Gal4 is strongly and preferentially expressed in MB $\gamma$ lobe neurons (Supplemental Fig. S1; Pfeiffer et al. 2008; Jenett et al. 2012). NP1131 and 1471 are preferentially expressed in $\gamma$ lobe, although their Gal4 expression in MB is not strong enough (Aso et al. 2009). Blocking the output from R92F10-Gal4 labeled neurons also severely interrupted the retrieval of aversive 3-h memory, while inhibiting its Gal4 activity in $\mathrm{MB}$ rescued this memory retrieval deficiency (Fig. 3B). Interrupting the neuronal transmission from NP1131 or 1471 labeled neurons also impaired aversive 3-h memory retrieval (Fig. 3D).

In conclusion, in $\mathrm{MB}$, aversive 3-h memory is retrieved through the combination of $\gamma$ lobe and $\alpha / \beta$ lobe neurons. The crucial role of $\gamma$ lobe in aversive early memory retrieval is confirmed.

Only $\gamma$ lobe neurons can support the Rutabaga-dependent aversive 3-h memory

Rutabaga is suggested to be the coincidence detector mediating olfactory associative memory formation (Tempel et al. 1983; Tully and Quinn 1985; Han et al. 1992; Levin et al. 1992; Zhong and Wu 1993; Zars et al. 2000a,b; McGuire et al. 2003; Mao et al.
2004; Tomchik and Davis 2009; Gervasi et al. 2010). Although the unconditional stimulus relayed by dopaminergic neurons is mediated by $\gamma$ lobe in MB (Aso et al. 2012; Burke et al. 2012; Liu et al. 2012; Qin et al. 2012; Waddell 2013), it has not been reported whether $\gamma$ lobe neurons alone could support the Rutabaga-dependent aversive 3-h memory (Blum et al. 2009). Since output from $\gamma$ lobe neurons is crucial for the retrieval of aversive 3-h memory (Figs. 2, 3), it is still possible that $\gamma$ lobe neurons can support the Rutabaga-dependent aversive 3-h memory, besides aversive immediate memory (Zars et al. 2000a; Akalal et al. 2006; Blum et al. 2009) and appetitive STM (Trannoy et al. 2011). $r u t^{2080}$ is a loss of function allele of the rutabaga gene (Han et al. 1992; Thum et al. 2007; Blum et al. 2009). Here, we restored the Rutabaga protein expression in rut ${ }^{2080}$ mutants driven by different MB Gal4s. Consistent with our speculation, restoring Rutabaga expression in 5-HT1B-Gal4 labeled neurons could sufficiently rescue the deficiency of aversive 3-h memory in rut ${ }^{2080} \mathrm{mu}$ tants (Fig. 4). Meanwhile, inhibiting its Gal4 activity in MB reversed this rescue phenotype (Fig. 4), which further testified the role of $\gamma$ lobe neurons in supporting aversive memory formation. In addition, $\mathrm{MB} \alpha^{\prime} / \beta^{\prime}$ lobe and $\alpha / \beta$ lobe neurons could not support the Rutabaga-dependent aversive 3-h memory (Fig. 4; Thum et al. 2007; Blum et al. 2009).

Therefore, $\gamma$ lobe neurons are the only MB substructure that could support the Rutabaga-dependent aversive 3-h memory. We revealed the consistency between aversive memory formation and retrieval. The Rutabaga-dependent aversive memory is originally formed or stored in $\mathrm{MB} \gamma$ lobe neurons. The retrieval of this memory requires the synaptic output from $\mathrm{MB} \gamma$ lobe besides recruiting the output from $\alpha / \beta$ lobes.

\section{Blocking the synaptic output from $\gamma$ lobe or $\alpha / \beta$ lobe neurons partially impairs the retrieval of appetitive 3 -h memory}

We then tested whether the retrieval of appetitive 3-h memory also required the output from both $\gamma$ lobe and $\alpha / \beta$ lobe neurons. Under the permissive temperature, all genotypes showed normal appetitive 3-h memory performances (Fig. 5A,C). Both 5-HT1BGal4/uas-shi and C739/+;uas-shi/+ flies showed partial deficiencies of appetitive 3-h memory retrieval under restrictive temperature (Fig. 5B). Besides, MB-Gal80 expression inhibited these memory retrieval deficiencies (Fig. 5D) which further testified the role of $\gamma$ lobe and $\alpha / \beta$ lobe neurons in appetitive early memory retrieval. In addition, 5-HT1B-Gal4/uas-shi and C739/+;uasshi/+ flies showed normal odor acuity and sugar response under both permissive and restrictive temperatures (Supplemental Table S1). Consistent with aversive 3-h memory retrieval (Figs. 2, 3; Krashes et al. 2007), blocking the output from $\alpha^{\prime} / \beta^{\prime}$ lobe

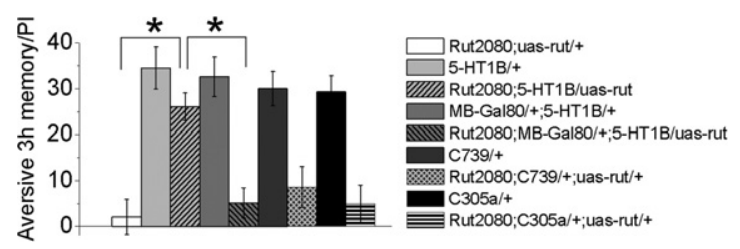

Figure 4. Only $\gamma$ lobe neurons could support the Rutabaga-dependent aversive 3-h memory in MB. The memory deficiency in rut ${ }^{2080}$ mutants could only be rescued by expressing uas-rut in 5-HT1B-Gal4 labeled $\gamma$ lobe neurons, but not in $\alpha / \beta$ lobe and $\alpha^{\prime} / \beta^{\prime}$ lobe neurons labeled by C739-Gal4 and C305a-Gal4. Inhibiting the Gal4 activity in MB by MB-Gal80 reversed the rescue phenotype driven by 5-HT1B-Gal4. In all groups, $N \geq 8$. All error bars indicate SEM. $\left(^{*}\right) P<0.05$, (PI) performance index. 

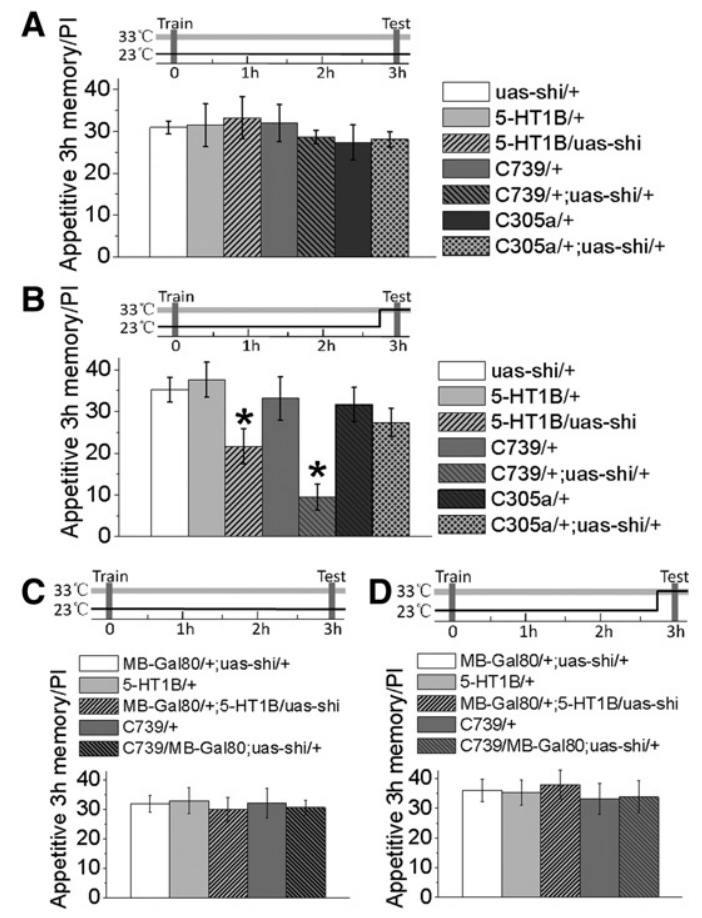

Figure 5. $\quad \gamma$ lobe and $\alpha / \beta$ lobe neurons are both required for the retrieval of appetitive 3-h memory. (A) When constantly under the permissive temperature, all genotypes showed normal appetitive 3-h memory performances. (B) Only blocking the output from 5-HT1B-Gal4 labeled $\gamma$ lobe or C739-Gal4 labeled $\alpha / \beta$ lobes impaired appetitive 3-h memory retrieval. (C) Appetitive 3-h memory performance was normal in all genotypes when constantly under the permissive temperature. (D) Inhibiting the Gal4 activity of 5-HT1B-Gal4 or C739-Gal4 in MB by MB-Gal80 rescued the deficiencies of appetitive 3-h memory retrieval. In all groups, $N \geq 8$. All error bars indicate SEM. $\left(^{*}\right) P<0.05$, (PI) performance index.

neurons did not affect appetitive 3-h memory retrieval (Fig. 5B; Trannoy et al. 2011). Interrupting the $\gamma$ lobe or $\alpha / \beta$ lobe output also impaired the retrieval of appetitive immediate memory (Supplemental Fig. S2B) and appetitive 1-h memory (Supplemental Fig. S4).

To further confirm the role of $\alpha / \beta$ lobe neurons in appetitive memory retrieval, we examined another two $\alpha / \beta$ lobe Gal4 lines: NP3061 and NP6024 (Tanaka et al. 2008; Aso et al. 2009). These two Gal4s specifically label $\alpha / \beta$ lobe neurons in MB (Tanaka et al. 2008; Aso et al. 2009). Acutely blocking the output from these two Gal4 labeled neurons also impaired the retrieval of appetitive 3-h memories (Fig. 6B).

In conclusion, the retrieval of appetitive 3-h memory also requires the synaptic outputs from both $\gamma$ lobe and $\alpha / \beta$ lobe neurons. The role of $\alpha / \beta$ lobe neurons in appetitive early memory retrieval is confirmed.

The retrieval of appetitive and aversive 3-h memories is completely abolished when the outputs from $\gamma$ lobe and $\alpha / \beta$ lobe neurons are blocked simultaneously

Blocking the output from $\gamma$ lobe neurons severely impaired the retrieval of aversive 3-h memory (Figs. 2, 3), but from either $\gamma$ lobe or $\alpha / \beta$ lobes just partially impaired the retrieval of appetitive 3-h memory (Figs. 5, 6). This may due to the possibility that the contribution of $\gamma$ lobe and $\alpha / \beta$ lobe neurons to appetitive early memory retrieval is additive so that $\gamma$ lobe or $\alpha / \beta$ lobe neurons alone could partially support the retrieval of appetitive memory. We therefore generated a driver line C739-Gal4; 5-HT1B-Gal4, which labeled both $\gamma$ lobe and $\alpha / \beta$ lobe neurons (Fig. 7A). As speculated, the retrieval of appetitive 3-h memory was completely abolished when the outputs from both $\gamma$ lobe and $\alpha / \beta$ lobe neurons were blocked simultaneously (Fig. 7B,C,F), which was statistically different with the phenotype of blocking the output from either $\gamma$ lobe or $\alpha / \beta$ lobe neurons (Fig. 7F). So did appetitive 1-h memory retrieval (Supplemental Figs. S5, S6). The abolishment of memory performance was also observed in aversive 3-h memory retrieval (Fig. 7D,E) and olfactory immediate memory tests (Supplemental Fig. S2).

\section{Discussion}

\section{Using at least two neuronal ensembles to retrieve aversive and appetitive memories}

Previous experiments found a single but different neuronal ensemble to retrieve aversive versus appetitive early memory in MB. $\alpha / \beta$ lobe neurons are reported to be necessary for aversive memory retrieval (McGuire et al. 2001; Krashes et al. 2007), whereas $\gamma$ lobe neurons appear to be indispensable for appetitive memory retrieval (Trannoy et al. 2011).

However, some experimental conditions may restrict our understanding to such a view. To examine the roles of $\gamma$ lobe in aversive memory retrieval, a strong $\gamma$ lobe-specific Gal4 line is still needed (Tanaka et al. 2008; Aso et al. 2009). For example, $201 y$ Gal4 only labels a limited portion of $\gamma$ lobe neurons and has nonspecific expression in $\mathrm{MB} \alpha / \beta$ lobe neurons (Aso et al. 2009). NP21-Gal4 has strong expression in olfactory projection neurons and many other brain areas outside MB (Kimura et al. 2005). If the $\gamma$ lobe Gal4 used labels $\mathrm{MB} \alpha / \beta$ lobe neurons and olfactory projection neurons, the retrieval of olfactory memories (McGuire et al. 2001; Krashes et al. 2007) or the transmission of olfactory information (Masse et al. 2009) can be affected.

Some differences in experimental conditions might make us reveal the crucial role of $\alpha / \beta$ lobe neurons in appetitive early memory retrieval. In the previous study, the association time of $\mathrm{CS}+$ and sucrose reward was $1 \mathrm{~min}$, which may lead to the relatively low performance index of appetitive early memory in experiments containing C739/uas-shi group (generally near or below $20 \%)$ in the previous study. The association duration of CS+ and sucrose is $2 \mathrm{~min}$ in our study. We produced relatively higher and more stable appetitive early memory performances (near or above $30 \%$ ) so that the statistical difference between the memory performances of C739/uas-shi group and control groups can be
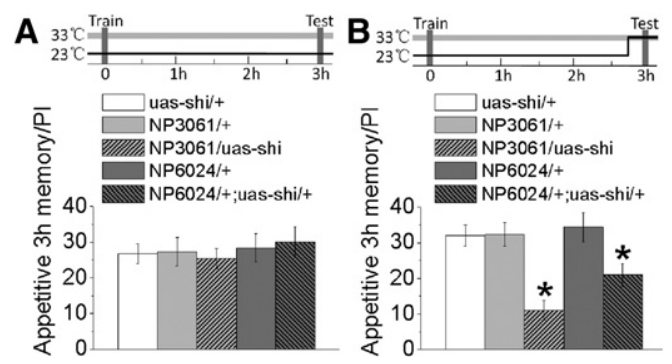

Figure 6. Interrupting the synaptic output from $\alpha / \beta$ lobe neurons driven by another two $\alpha / \beta$ lobe Gal $4 \mathrm{~s}$ also impairs the retrieval of appetitive 3-h memory. (A) Under permissive temperature, all genotypes showed normal appetitive 3-h memory performances. (B) When blocking the output from NP3061 or NP6024 labeled $\alpha / \beta$ lobe neurons, the retrieval of appetitive 3-h memory was also impaired. In all groups, $N \geq 8$. All error bars indicate SEM. $\left({ }^{*}\right) P<0.05$, $(\mathrm{PI})$ performance index. 
A
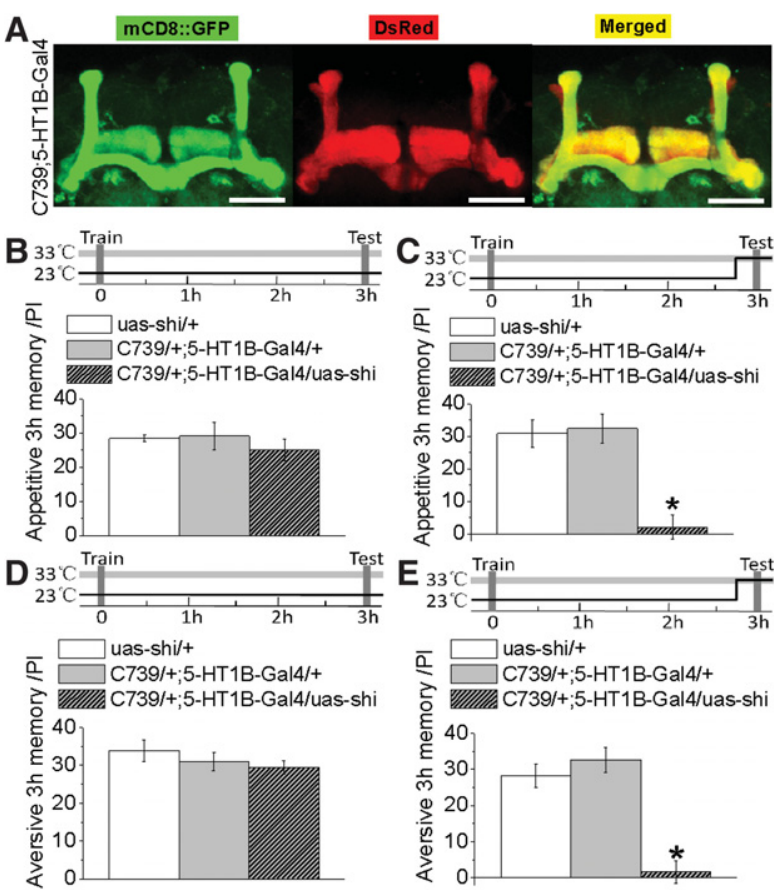

$\overline{0} \frac{1}{0} \frac{1}{1 h}$
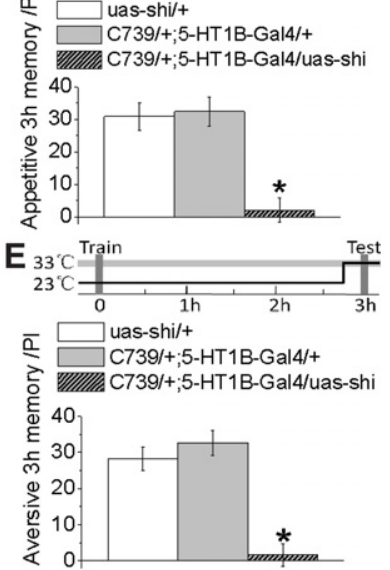

$\mathbf{F}$
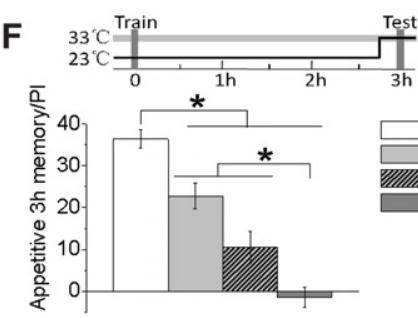

Figure 7. Blocking the outputs from $\gamma$ lobe and $\alpha / \beta$ lobe neurons simultaneously completely abolishes the retrieval of appetitive and aversive 3-h memories. (A) The expression pattern of mCD8::GFP denoted that C739;5-HT1B-Gal4 specifically labeled MB $\gamma$ lobe and $\alpha / \beta$ lobes, but not $\alpha^{\prime} / \beta^{\prime}$ lobes. (B) When constantly under the permissive temperature, all genotypes showed normal appetitive 3-h memory performances. (C) Appetitive 3-h memory performance was reduced to near zero when the neuronal transmissions from $\gamma$ lobe and $\alpha / \beta$ lobes were disrupted simultaneously in C739/+;5-HT1B-Gal4/uas-shi flies. (D) All genotypes showed normal aversive 3-h memory performances under the permissive temperature. (E) Aversive 3-h memory retrieval was completely abolished when blocking the outputs from $\gamma$ lobe and $\alpha / \beta$ lobes simultaneously in C739/+;5-HT1B-Gal4/uas-shi flies. ( F) Appetitive 3-h memory performance was statistically lower when inhibiting the outputs from $\gamma$ lobe and $\alpha / \beta$ lobe neurons simultaneously, compared with blocking the output from either $\gamma$ lobe or $\alpha / \beta$ lobe neurons. Scale bar, $50 \mu \mathrm{m}$. In all groups, $N \geq 8$. All error bars indicate SEM. $\left(^{*}\right) P<0.05$, (PI) performance index.

uncovered. Besides, our study used the uas-shi fly strain with two copy insertions rather than the one with only one copy insertion in the previous study (Trannoy et al. 2011). The two copy insertion uas-shi line generally has a better effect on blocking the neuronal transmission than the one copy insertion uas-shi line (Schwaerzel et al. 2002) so that the necessity of $\alpha / \beta$ lobe neurons in appetitive memory retrieval could be revealed using the two copy insertion uas-shi line. In addition, we used another two $\alpha /$ $\beta$ lobe Gal4 lines to exam and confirm the role of $\alpha / \beta$ lobe neurons in appetitive memory retrieval (Fig. 6) even though it was not detected by just using C739 in the previous study. Blocking the synaptic outputs from these two Gal4 labeled neurons also impaired appetitive early memory retrieval (Fig. 6).
In our study, using Gal4 lines preferentially labeling $\gamma$ lobe or $\alpha / \beta$ lobes, we revealed a crucial role of MB $\gamma$ lobe neurons in aversive intermediate-term memory retrieval (Figs 2,3 ) and $\alpha / \beta$ lobe neurons in appetitive intermediate-term memory retrieval (Figs. 5-7). Inhibiting the Gal4 activity in MB by expressing MB-Gal80 further testified the role of $\gamma$ lobe and $\alpha / \beta$ lobes in olfactory memory retrieval. Besides intermediate-term memory, $\gamma$ lobe and $\alpha / \beta$ lobes are both necessary for retrieving aversive and appetitive STMs (Supplemental Figs. S2-S4). These discoveries are consistent with a recent study (Cervantes-Sandoval et al. 2013). In accordance with previous studies, $\alpha^{\prime} / \beta^{\prime}$ lobe neurons are dispensable for the retrieval of aversive 3-h memory (Krashes et al. 2007; Cervantes-Sandoval et al. 2013) and appetitive early memory (Trannoy et al. 2011). A variance on the observation of $\alpha^{\prime} / \beta^{\prime}$ lobe neurons in appetitive early memory retrieval in another study can be explained by some differences in experimental conditions discussed in that study (CervantesSandoval et al. 2013), as well as the odor concentrations and training parameters.

The functions of different MB substructures are further confirmed now. Thus, our study and previous experiments together suggest a new view of how memory is retrieved in $\mathrm{MB}$, the olfactory learning and memory center in Drosophila. Both aversive and appetitive early memories are retrieved through at least two neuronal ensembles in MB: the combination of $\gamma$ lobe and $\alpha / \beta$ lobe neurons.

It has been shown that MB may have common origins with vertebrate pallium (Tomer et al. 2010). Recently, accumulating studies on mammalian neural circuits began to reveal that mammalian brain also uses two neuronal ensembles to retrieve certain memories (Sepulcre et al. 2008; Takahashi et al. 2008; Goshen et al. 2011; Izumi et al. 2011; Suzuki and Naya 2011). Therefore, our study implies that using at least two neuronal ensembles to retrieve memory may be an evolutionarily conserved strategy across various species during memory processing.

In the anterior part of $\mathrm{MB}$, the axons of $\mathrm{MB} \gamma$ lobe and $\beta$ lobe neurons are horizontally located, while the axons of $\mathrm{MB} \alpha$ lobe neurons are vertically located (Tanaka et al. 2008; Aso et al. 2009). Several evidences indicate that there may be some functional dissociation between these spatially differentiated lobes (Pascual and Preat 2001; Hu et al. 2010). These complexities imply that $\mathrm{MB} \gamma$ lobe and $\alpha / \beta$ lobes may connect to diverse downstream circuits and regulatory circuits during memory retrieval.

\section{Olfactory early memories: from formation to retrieval}

During the formation of aversive olfactory memory, the unconditional dopaminergic input is mediated by $\gamma$ lobe in MB (Qin et al. 2012). Rutabaga serves as a coincidence detector mediating the formation of olfactory associative memory by associating the olfactory information with unconditional stimuli (Tempel et al. 1983; Tully and Quinn 1985; Han et al. 1992; Levin et al. 1992; Zhong and Wu 1993; Zars et al. 2000a,b; Mao et al. 2004; Tomchik and Davis 2009; Gervasi et al. 2010). The Rutabaga rescue experiments indicate that aversive STM is formed in MB $\gamma$ lobe (Fig. 3; Zars et al. 2000a; Blum et al. 2009), while the appetitive early memory is formed in both $\mathrm{MB} \gamma$ lobe and olfactory projection neurons (Thum et al. 2007; Trannoy et al. 2011). Our study further confirmed that aversive ITM is formed and stored in MB $\gamma$ lobe neurons (Fig. 4). Subsequently, these olfactory memories may be consolidated by the recurrent activity of $\mathrm{MB} \alpha^{\prime} / \beta^{\prime}$ neuron-DPM/APL neuron loop (Keene et al. 2006; Krashes et al. 2007; Lee et al. 2011; Pitman et al. 2011; Wu et al. 2011; Cervantes-Sandoval and Davis 2012; Qin et al. 2012).

Thus, the current study leads us to rethink the information flow of memory processing in Drosophila from memory formation 
to memory retrieval. Before memory retrieval, aversive memory formed in $\gamma$ lobe neurons is at least not fully transferred to $\alpha / \beta$ lobe neurons, since $\gamma$ lobe output is still needed during aversive memory retrieval (Fig. 2) and $\gamma$ lobe neurons can support the Rutabaga-dependent aversive 3-h memory (Fig. 4). Appetitive memory formed in $\mathrm{MB} \gamma$ lobe and olfactory projection neurons is not completely retrieved through $\gamma$ lobe, but through the combination of $\gamma$ lobe and $\alpha / \beta$ lobes (Figs. $5-7$ ).

Although aversive and appetitive STMs are mainly not formed in $\alpha / \beta$ lobes, $\alpha / \beta$ lobes are recruited during the process of memory retrieval. It is possible that there may be some communication between $\gamma$ lobe neurons and $\alpha / \beta$ lobe neurons before or during memory retrieval. This communication can be mediated by $\mathrm{MB}$ intrinsic neurons and extrinsic neurons (Krashes et al. 2007; Lee et al. 2011; Qin et al. 2012), or achieved by direct interaction (Christiansen et al. 2011).

\section{Differentiated usage of $\gamma$ lobe output during the retrieval of aversive and appetitive 3-h memories}

Blocking the synaptic output from $\gamma$ lobe during retrieval severely impaired aversive 3-h memory, but just partially impaired appetitive 3-h memory. Two possible reasons may account for the differentiated usage of $\gamma$ lobe output: multiple memory traces and downstream neural circuits.

Aversive early memory is mainly formed in MB $\gamma$ lobe (Fig. 4; Blum et al. 2009; Qin et al. 2012). Thus, $\gamma$ lobe output is indispensable for retrieving aversive memory (Fig. 2). But $\gamma$ lobe output is just partially dispensable for retrieving appetitive memory. This may be because the appetitive memory stored in olfactory projection neurons can still be retrieved through the output from $\alpha / \beta$ lobes when the memory stored in $\mathrm{MB} \gamma$ lobe neurons cannot be retrieved through the $\gamma$ lobe output. Therefore, only simultaneously blocking the outputs from $\gamma$ lobe and $\alpha / \beta$ lobes could completely interrupt the retrieval of appetitive 3-h memory (Fig. 7).

Another possibility is that the downstream neurons of $\mathrm{MB}$ may use different strategies to integrate the information from $\gamma$ lobe and $\alpha / \beta$ lobes. Compared with the appetitive memory downstream, the activation of aversive memory downstream may rely more on the information from $\gamma$ lobe output. In addition, the activation thresholds may differ in these two downstream circuits. The activation threshold of aversive memory downstream circuits may be higher than appetitive memory downstream circuits so that more signals from $\mathrm{MB}$ are needed to activate the aversive memory downstream circuits efficiently. Unfortunately, the exact downstream circuits of $\mathrm{MB}$ remain unclear, although some neurons affecting the retrieval of certain olfactory memories were identified recently (Krashes et al. 2009; Liu and Davis 2009; Waddell 2010; Sejourne et al. 2011; Chen et al. 2012; Pai et al. 2013). With the progress on neural circuit studies in Drosophila (Pfeiffer et al. 2008; Tanaka et al. 2008; Aso et al. 2009; Chiang et al. 2011; del Valle Rodriguez et al. 2012), this possibility may be tested in the future.

In conclusion, our studies characterized the MB substructures involved in the retrieval of aversive and appetitive early memories. On this basis, our study and previous experiments together suggest a new view of how olfactory memories are retrieved when considering the MB substructures involved in memory formation together. In MB, the Drosophila learning and memory center, aversive and appetitive early memories are retrieved through the outputs from both $\gamma$ lobe and $\alpha / \beta$ lobes. The discovery of using at least two neuronal ensembles to retrieve memory leads us to rethink how olfactory memories are processed from memory formation to retrieval. More broadly, this discovery implies that the manner of using two neuronal ensembles to retrieve memory may serve as an evolutionarily conserved strategy during memory processing across various species.

\section{Materials and Methods}

\section{Fly stocks and culture}

Flies were raised at $23^{\circ} \mathrm{C}, 50 \%$ humidity on standard medium. 5-HT1B-Gal4 and R92F10-Gal4 were obtained from Bloomington Drosophila Stock Center. C739, C305a, and 1471 lines were kindly provided by K. Ito. uas-shi (with two copy insertions [Kitamoto 2001]) and uas-mCD8::GFP;MB247-DsRed (Lee and Luo 1999; Riemensperger et al. 2005) lines were gifts from Cold Spring Harbor Laboratory. rut ${ }^{2080}$; uas-rut flies were a gift from J. Dubnau. MB-Gal80 flies were kindly provided by S. Waddell. NP1131, NP6024, and NP3061 were provided by Drosophila Genetic Resource Center (DGRC). All the Gal4 and Gal80 lines were outcrossed with the wild-type strain $w^{111}$ (isoCJ1) for five generations. We generated MB-Gal80; uas-shi, MB-Gal80;5-HT1BGal4, and C739-Gal4;5-HT1B-Gal4 lines ourselves. Unless specified, all the flies tested were a mixed population of sexes. rut ${ }^{2080}$ and NP6024 alleles both locate on the first chromosome. In $r u t^{2080}$ rescue experiments, only male hemizygotes were counted. Only females were counted in experiments with NP6024/+;uasshi $/+$ flies.

\section{Behavioral analysis}

Olfactory aversive conditioning was performed as described previously (Tully and Quinn 1985). Briefly, about 100 flies successively experienced 90-sec air, 1-min CS + odor and electric shock, 45-sec air, 1-min CS - odor without electric shock, and 45-sec air, in a training tube covered internally with copper grid. The two odors used for behavior analysis were 3-octanol $(0.15 \% \mathrm{v} / \mathrm{v})$ and 4 methylcyclohexanol $(0.10 \% \mathrm{v} / \mathrm{v}$; Sigma-Aldrich Co. LLC). The 1 -min electric shock was delivered as 12 cycles ( $60 \mathrm{~V}$ for $1.5 \mathrm{sec}$ and subsequent rest for $3.5 \mathrm{sec}$ ). $60 \mathrm{~V}$ is a commonly used voltage for electric shock (Yin et al. 1994; Pagani et al. 2009; Shuai et al. 2010; Qin et al. 2012). All the flies were trained, kept, or tested at $23^{\circ} \mathrm{C}$ or $33^{\circ} \mathrm{C}$ with $70 \%$ relative humidity in dark dimly red light. If temperature shift was needed, flies were accustomed to the new temperature $15 \mathrm{~min}$ ahead. A half performance index (PI) value is calculated as follows using the fly numbers choosing CS + or CS - odors: $(\mathrm{CS}--\mathrm{CS}+) /(\mathrm{CS}-+\mathrm{CS}+) \times 100 \%$. A single PI value is the average of two half PIs with opposite CS+ odors.

Olfactory appetitive conditioning was performed similar to the previously described protocol (Keene et al. 2006). Briefly, about 100 flies successively experienced 20-sec air, 2-min CSodor, 1-min air in a training tube without sugar, and then 2-min CS+ odor, 20-sec air in a training tube covered internally with dried sucrose paper. All the flies were transferred to new food vials $24 \mathrm{~h}$ before starvation, and then starved for $\sim 36 \mathrm{~h}$ at $23^{\circ} \mathrm{C}$ before training. A half PI value is calculated as $(\mathrm{CS}+-\mathrm{CS}-) /(\mathrm{CS}-+$ $\mathrm{CS}+) \times 100 \%$. Other conditions were the same as for aversive conditioning.

\section{Sensory acuity tests}

\section{Odor acuity}

Odors were the same as for olfactory conditioning. Flies were allowed to choose between each odor and air in a T-maze for $2 \mathrm{~min}$.

\section{Shock avoidance}

Electric shocks were delivered the same as in aversive conditioning. Flies were allowed to choose between the arm with electric shock and the arm without electric shock in the T-maze for $1 \mathrm{~min}$.

\section{Sugar acuity}

Flies were allowed to choose between the arm covered with dried sucrose paper and the arm without sugar in the T-maze for $2 \mathrm{~min}$. 


\section{Gal4 expression pattern analysis}

We crossed uas-mCD8::GFP;MB247-dsRed virgin female flies with the male flies of each Gal4 line to generate the genotypes for Gal4 expression pattern analysis. The first generation female offspring were dissected at 3- to 5-d old. The dissected central brains were fixed in $4 \%$ paraformaldehyde (in PBS) for $15 \mathrm{~min}$, and then rinsed in PBS $\left(137 \mathrm{mM} \mathrm{NaCl}, 2.7 \mathrm{mM} \mathrm{KCl}, 10 \mathrm{mM} \mathrm{Na}_{2} \mathrm{HPO}_{4}, 2 \mathrm{mM}\right.$ $\left.\mathrm{KH}_{2} \mathrm{PO}_{4}, \mathrm{pH}=7.4\right)$ three times. They were then incubated in VECTASHIELD Mounting Medium (Vector Laboratories, Inc.). Confocal analysis was performed on a Zeiss LSM 710 confocal microscope. Confocal stacks were then processed using Imaris (Bitplane AG).

\section{Statistics}

In all sensory acuity tests, $N \geq 6$. In all other behavioral experiments, $N \geq 8$. Statistical analyses were performed using Tukey's post hoc tests following one-way ANOVA (set significance level as 0.05). If not specified, asterisks denote statistically significant differences between the experimental groups and their unmarked control groups $(P<0.05)$. All error bars indicate standard error of the mean (SEM).

\section{Acknowledgments}

We thank Bloomington Drosophila Stock Center, Drosophila Genetic Resource Center, Cold Spring Harbor Laboratory, Kei Ito, Josh Dubnau, and Scott Waddell for providing us with fly stocks. This work was supported by grants from Program 973 of the Ministry of Science and Technology of China (2006CB 500806, 2009CB941301, and 2013CB835102), Tsinghua University Initiative Scientific Research Program (20111080956), scientific project for advisor of Beijing excellent doctoral dissertation (20111000307), and a grant from the Tsinghua-Yue-Yuen Medical Sciences Fund (to Y.Z.).

\section{References}

Akalal DB, Wilson CF, Zong L, Tanaka NK, Ito K, Davis RL. 2006. Roles for Drosophila mushroom body neurons in olfactory learning and memory. Learn Mem 13: 659-668.

Aso Y, Grubel K, Busch S, Friedrich AB, Siwanowicz I, Tanimoto H. 2009. The mushroom body of adult Drosophila characterized by GAL4 drivers. J Neurogenet 23: 156-172.

Aso Y, Herb A, Ogueta M, Siwanowicz I, Templier T, Friedrich AB, Ito K, Scholz H, Tanimoto H. 2012. Three dopamine pathways induce aversive odor memories with different stability. PLoS Genet 8: e1002768.

Bellen HJ, Tong C, Tsuda H. 2010. 100 years of Drosophila research and its impact on vertebrate neuroscience: A history lesson for the future. Nat Rev Neurosci 11: 514-522.

Berry JA, Cervantes-Sandoval I, Nicholas EP, Davis RL. 2012. Dopamine is required for learning and forgetting in Drosophila. Neuron 74: 530-542.

Blum AL, Li W, Cressy M, Dubnau J. 2009. Short- and long-term memory in Drosophila require cAMP signaling in distinct neuron types. Curr Biol 19: $1341-1350$.

Buchanan ME, Davis RL. 2010. A distinct set of Drosophila brain neurons required for neurofibromatosis type 1-dependent learning and memory. J Neurosci 30: 10135-10143.

Burke CJ, Huetteroth W, Owald D, Perisse E, Krashes MJ, Das G, Gohl D, Silies M, Certel S, Waddell S. 2012. Layered reward signalling through octopamine and dopamine in Drosophila. Nature 492: 433-437.

Cervantes-Sandoval I, Davis RL. 2012. Distinct traces for appetitive versus aversive olfactory memories in DPM neurons of Drosophila. Curr Biol 22: $1247-1252$.

Cervantes-Sandoval I, Martin-Pena A, Berry JA, Davis RL. 2013. System-like consolidation of olfactory memories in Drosophila. J Neurosci 33: 9846-9854.

Chen CC, Wu JK, Lin HW, Pai TP, Fu TF, Wu CL, Tully T, Chiang AS. 2012. Visualizing long-term memory formation in two neurons of the Drosophila brain. Science 335: 678-685.

Chiang AS, Lin CY, Chuang CC, Chang HM, Hsieh CH, Yeh CW, Shih CT, Wu JJ, Wang GT, Chen YC, et al. 2011. Three-dimensional reconstruction of brain-wide wiring networks in Drosophila at single-cell resolution. Curr Biol 21: 1-11.
Christiansen F, Zube C, Andlauer TF, Wichmann C, Fouquet W, Owald D, Mertel S, Leiss F, Tavosanis G, Farca Luna AJ, et al. 2011. Presynapses in kenyon cell dendrites in the mushroom body calyx of Drosophila. J Neurosci 31: 9696-9707.

Davis RL. 2005. Olfactory memory formation in Drosophila: From molecular to systems neuroscience. Annu Rev Neurosci 28: 275-302.

del Valle Rodriguez A, Didiano D, Desplan C. 2012. Power tools for gene expression and clonal analysis in Drosophila. Nat Methods 9: $47-55$.

Gervasi N, Tchenio P, Preat T. 2010. PKA dynamics in a Drosophila learning center: Coincidence detection by rutabaga adenylyl cyclase and spatial regulation by dunce phosphodiesterase. Neuron 65: 516-529.

Goshen I, Brodsky M, Prakash R, Wallace J, Gradinaru V, Ramakrishnan C, Deisseroth K. 2011. Dynamics of retrieval strategies for remote memories. Cell 147: 678-689.

Han PL, Levin LR, Reed RR, Davis RL. 1992. Preferential expression of the Drosophila rutabaga gene in mushroom bodies, neural centers for learning in insects. Neuron 9: 619-627.

Hu A, Zhang W, Wang Z. 2010. Functional feedback from mushroom bodies to antennal lobes in the Drosophila olfactory pathway. Proc Natl Acad Sci 107: 10262-10267.

Huang C, Zheng X, Zhao H, Li M, Wang P, Xie Z, Wang L, Zhong Y. 2012. A permissive role of mushroom body $\alpha / \beta$ core neurons in long-term memory consolidation in Drosophila. Curr Biol 22: 1981-1989.

Izumi T, Boku S, Shinmin W, Inoue T, Konno K, Yamaguchi T, Yoshida T, Matsumoto M, Watanabe M, Koyama T, et al. 2011. Retrieval of conditioned fear activates the basolateral and intercalated nucleus of amygdala. J Neurosci Res 89: 773-790.

Jenett A, Rubin GM, Ngo TT, Shepherd D, Murphy C, Dionne H, Pfeiffer BD, Cavallaro A, Hall D, Jeter J, et al. 2012. A GAL4-driver line resource for Drosophila neurobiology. Cell Rep 2: 991-1001.

Kasuya J, Ishimoto H, Kitamoto T. 2009. Neuronal mechanisms of learning and memory revealed by spatial and temporal suppression of neurotransmission using shibire ${ }^{\mathrm{ts} 1}$, a temperature-sensitive dynamin mutant gene in Drosophila melanogaster. Front Mol Neurosci 2: 11.

Keene AC, Waddell S. 2007. Drosophila olfactory memory: Single genes to complex neural circuits. Nat Rev Neurosci 8: 341-354.

Keene AC, Krashes MJ, Leung B, Bernard JA, Waddell S. 2006. Drosophila dorsal paired medial neurons provide a general mechanism for memory consolidation. Curr Biol 16: 1524-1530.

Kim Y-T, Wu C-F. 1990. Allelic interactions at the shibire locus of Drosophila: Effects on behavior. J Neurogenet 7: 1-14.

Kimura K, Ote M, Tazawa T, Yamamoto D. 2005. Fruitless specifies sexually dimorphic neural circuitry in the Drosophila brain. Nature 438: 229-233.

Kitamoto T. 2001. Conditional modification of behavior in Drosophila by targeted expression of a temperature-sensitive shibire allele in defined neurons. J Neurobiol 47: 81-92.

Krashes MJ, Keene AC, Leung B, Armstrong JD, Waddell S. 2007. Sequential use of mushroom body neuron subsets during Drosophila odor memory processing. Neuron 53: 103-115.

Krashes MJ, DasGupta S, Vreede A, White B, Armstrong JD, Waddell S. 2009. A neural circuit mechanism integrating motivational state with memory expression in Drosophila. Cell 139: 416-427.

Kuhlmann S, Piel M, Wolf OT. 2005. Impaired memory retrieval after psychosocial stress in healthy young men. J Neurosci 25: 2977-2982.

Lee T, Luo L. 1999. Mosaic analysis with a repressible cell marker for studies of gene function in neuronal morphogenesis. Neuron 22: 451-461.

Lee PT, Lin HW, Chang YH, Fu TF, Dubnau J, Hirsh J, Lee T, Chiang AS. 2011. Serotonin-mushroom body circuit modulating the formation of anesthesia-resistant memory in Drosophila. Proc Natl Acad Sci 108: 13794-13799.

Levin LR, Han PL, Hwang PM, Feinstein PG, Davis RL, Reed RR. 1992. The Drosophila learning and memory gene rutabaga encodes a $\mathrm{Ca}^{2+} /$ calmodulin-responsive adenylyl cyclase. Cell 68: 479-489.

Liu X, Davis RL. 2009. The GABAergic anterior paired lateral neuron suppresses and is suppressed by olfactory learning. Nat Neurosci 12: $53-59$.

Liu C, Placais PY, Yamagata N, Pfeiffer BD, Aso Y, Friedrich AB, Siwanowicz I, Rubin GM, Preat T, Tanimoto H. 2012. A subset of dopamine neurons signals reward for odour memory in Drosophila. Nature 488: 512-516.

Mao Z, Roman G, Zong L, Davis RL. 2004. Pharmacogenetic rescue in time and space of the rutabaga memory impairment by using Gene-Switch. Proc Natl Acad Sci 101: 198-203.

Margulies C, Tully T, Dubnau J. 2005. Deconstructing memory in Drosophila. Curr Biol 15: R700-R713.

Masse NY, Turner GC, Jefferis GS. 2009. Olfactory information processing in Drosophila. Curr Biol 19: R700-R713.

McGuire SE, Le PT, Davis RL. 2001. The role of Drosophila mushroom body signaling in olfactory memory. Science 293: 1330-1333. 
McGuire SE, Le PT, Osborn AJ, Matsumoto K, Davis RL. 2003. Spatiotemporal rescue of memory dysfunction in Drosophila. Science 302: $1765-1768$.

Pagani MR, Oishi K, Gelb BD, Zhong Y. 2009. The phosphatase SHP2 regulates the spacing effect for long-term memory induction. Cell 139: $186-198$.

Pai TP, Chen CC, Lin HH, Chin AL, Lai JS, Lee PT, Tully T, Chiang AS. 2013. Drosophila ORB protein in two mushroom body output neurons is necessary for long-term memory formation. Proc Natl Acad Sci 110: $7898-7903$.

Pascual A, Preat T. 2001. Localization of long-term memory within the Drosophila mushroom body. Science 294: 1115-1117.

Pfeiffer BD, Jenett A, Hammonds AS, Ngo TT, Misra S, Murphy C, Scully A, Carlson JW, Wan KH, Laverty TR, et al. 2008. Tools for neuroanatomy and neurogenetics in Drosophila. Proc Natl Acad Sci 105: $9715-9720$.

Pitman JL, Huetteroth W, Burke CJ, Krashes MJ, Lai SL, Lee T, Waddell S. 2011. A pair of inhibitory neurons are required to sustain labile memory in the Drosophila mushroom body. Curr Biol 21: 855-861.

Qin H, Cressy M, Li W, Coravos JS, Izzi SA, Dubnau J. 2012. $\gamma$ neurons mediate dopaminergic input during aversive olfactory memory formation in Drosophila. Curr Biol 22: 608-614.

Riemensperger T, Voller T, Stock P, Buchner E, Fiala A. 2005. Punishment prediction by dopaminergic neurons in Drosophila. Curr Biol 15: 1953-1960.

Schwaerzel M, Heisenberg M, Zars T. 2002. Extinction antagonizes olfactory memory at the subcellular level. Neuron 35: 951-960.

Sejourne J, Placais PY, Aso Y, Siwanowicz I, Trannoy S, Thoma V, Tedjakumala SR, Rubin GM, Tchenio P, Ito K, et al. 2011. Mushroom body efferent neurons responsible for aversive olfactory memory retrieval in Drosophila. Nat Neurosci 14: 903-910.

Sepulcre J, Masdeu JC, Sastre-Garriga J, Goni J, Velez-de-Mendizabal N, Duque B, Pastor MA, Bejarano B, Villoslada P. 2008. Mapping the brain pathways of declarative verbal memory: Evidence from white matter lesions in the living human brain. Neuroimage 42: $1237-1243$.

Shuai Y, Lu B, Hu Y, Wang L, Sun K, Zhong Y. 2010. Forgetting is regulated through Rac activity in Drosophila. Cell 140: 579-589.

Silva AJ, Zhou Y, Rogerson T, Shobe J, Balaji J. 2009. Molecular and cellular approaches to memory allocation in neural circuits. Science 326: $391-395$

Small SA, Schobel SA, Buxton RB, Witter MP, Barnes CA. 2011. A pathophysiological framework of hippocampal dysfunction in ageing and disease. Nat Rev Neurosci 12: 585-601.

Suzuki W, Naya Y. 2011. Two routes for remembering the past. Cell 147: $493-495$.

Takahashi E, Ohki K, Kim DS. 2008. Dissociated pathways for successful memory retrieval from the human parietal cortex: Anatomical and functional connectivity analyses. Cereb Cortex 18: 1771-1778.

Tanaka NK, Tanimoto H, Ito K. 2008. Neuronal assemblies of the Drosophila mushroom body. J Comp Neurol 508: 711-755.
Tempel BL, Bonini N, Dawson DR, Quinn WG. 1983. Reward learning in normal and mutant Drosophila. Proc Natl Acad Sci 80: $1482-1486$.

Thum AS, Jenett A, Ito K, Heisenberg M, Tanimoto H. 2007. Multiple memory traces for olfactory reward learning in Drosophila. J Neurosci 27: $11132-11138$.

Tomchik SM, Davis RL. 2009. Dynamics of learning-related cAMP signaling and stimulus integration in the Drosophila olfactory pathway. Neuron 64: $510-521$.

Tomer R, Denes AS, Tessmar-Raible K, Arendt D. 2010. Profiling by image registration reveals common origin of annelid mushroom bodies and vertebrate pallium. Cell 142: 800-809.

Trannoy S, Redt-Clouet C, Dura JM, Preat T. 2011. Parallel processing of appetitive short- and long-term memories in Drosophila. Curr Biol 21: $1647-1653$.

Tully T, Quinn WG. 1985. Classical conditioning and retention in normal and mutant Drosophila melanogaster. J Comp Physiol A 157: 263-277.

Tye KM, Deisseroth K. 2012. Optogenetic investigation of neural circuits underlying brain disease in animal models. Nat Rev Neurosci 13: 251-266.

van Strien NM, Cappaert NL, Witter MP. 2009. The anatomy of memory: An interactive overview of the parahippocampal-hippocampal network. Nat Rev Neurosci 10: 272-282.

Waddell S. 2010. Dopamine reveals neural circuit mechanisms of fly memory. Trends Neurosci 33: 457-464.

Waddell S. 2013. Reinforcement signalling in Drosophila; dopamine does it all after all. Curr Opin Neurobiol 23: 324-329.

Wu CL, Shih MF, Lai JS, Yang HT, Turner GC, Chen L, Chiang AS. 2011. Heterotypic gap junctions between two neurons in the Drosophila brain are critical for memory. Curr Biol 21: 848-854.

Yin JC, Wallach JS, Del Vecchio M, Wilder EL, Zhou H, Quinn WG, Tully T. 1994. Induction of a dominant negative CREB transgene specifically blocks long-term memory in Drosophila. Cell 79: 49-58.

Yu D, Akalal DB, Davis RL. 2006. Drosophila $\alpha / \beta$ mushroom body neurons form a branch-specific, long-term cellular memory trace after spaced olfactory conditioning. Neuron 52: 845-855.

Yuan Q, Lin F, Zheng X, Sehgal A. 2005. Serotonin modulates circadian entrainment in Drosophila. Neuron 47: 115-127.

Zars T. 2010. Short-term memories in Drosophila are governed by general and specific genetic systems. Learn Mem 17: 246-251.

Zars T, Fischer M, Schulz R, Heisenberg M. 2000a. Localization of a short-term memory in Drosophila. Science 288: 672-675.

Zars T, Wolf R, Davis R, Heisenberg M. 2000b. Tissue-specific expression of a type I adenylyl cyclase rescues the rutabaga mutant memory defect: In search of the engram. Learn Mem 7: 18-31.

Zhong Y, Wu CF. 1993. Differential modulation of potassium currents by cAMP and its long-term and short-term effects: Dunce and rutabaga mutants of Drosophila. J Neurogenet 9: 15-27.

Received May 23, 2013; accepted in revised form July 10, 2013. 


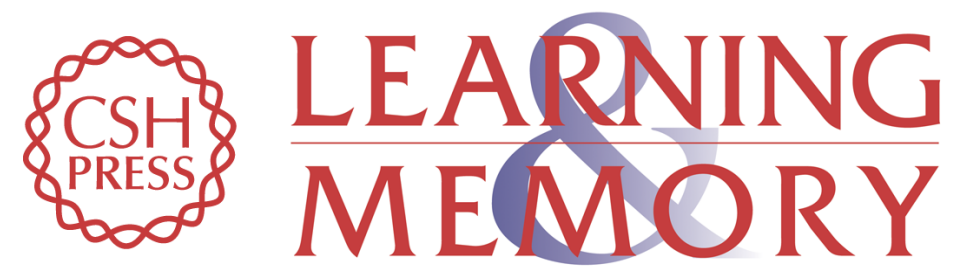

\title{
Requirement of the combination of mushroom body $\gamma$ lobe and $\alpha / \beta$ lobes for the retrieval of both aversive and appetitive early memories in Drosophila
}

\author{
Zhiyong Xie, Cheng Huang, Bo Ci, et al.
}

Learn. Mem. 2013, 20:

Access the most recent version at doi:10.1101/lm.031823.113

\section{Supplemental http://learnmem.cshlp.org/content/suppl/2013/08/05/20.9.474.DC1 Material}

References This article cites 75 articles, 20 of which can be accessed free at: http://learnmem.cshlp.org/content/20/9/474.full.html\#ref-list-1

Creative This article is distributed exclusively by Cold Spring Harbor Laboratory Press for the Commons first 12 months after the full-issue publication date (see License http://learnmem.cshlp.org/site/misc/terms.xhtml). After 12 months, it is available under a Creative Commons License (Attribution-NonCommercial 3.0 Unported), as described at http://creativecommons.org/licenses/by-nc/3.0/.

Email Alerting Receive free email alerts when new articles cite this article - sign up in the box at the Service top right corner of the article or click here. 